\title{
Quantifying Data Locality in Dynamic Parallelism in GPUs
}

\author{
Xulong Tang \\ Pennsylvania State University \\ State College, PA, USA \\ xzt102@psu.edu \\ Adwait Jog \\ College of William \& Mary \\ Williamsburg, VA, USA \\ ajog@wm.edu
}

\author{
Ashutosh Pattnaik \\ Pennsylvania State University \\ State College, PA, USA \\ ashutosh@psu.edu
Mahmut Taylan Kandemir
Pennsylvania State University
State College, PA, USA
mtk2@psu.edu

\author{
Onur Kayiran \\ Advanced Micro Devices, Inc. \\ Santa Clara, CA, USA \\ onur.kayiran@amd.com \\ Chita Das \\ Pennsylvania State University \\ State College, PA, USA \\ cxd12@psu.edu
}

\begin{abstract}
Dynamic parallelism (DP) is a new feature of emerging GPUs that allows new kernels to be generated and scheduled from the deviceside (GPU) without the host-side (CPU) intervention. To efficiently support DP, one of the major challenges is to saturate the GPU processing elements and provide them with the required data in a timely fashion. In this paper, we first conduct a limit study on the performance improvements that can be achieved by hardware schedulers that are provided with accurate data reuse information. We next propose LASER, a Locality-Aware SchedulER, where the hardware schedulers employ data reuse monitors to help make scheduling decisions to improve data locality at runtime. Experimental results on 16 benchmarks show that LASER, on an average, can improve performance by $11.3 \%$.
\end{abstract}

\section{CCS CONCEPTS}

- Computing methodologies $\rightarrow$ Massively parallel and highperformance simulations; • Computer systems organization $\rightarrow$ Single instruction, multiple data;

\section{KEYWORDS}

GPGPU; data reuse; performance evaluation

\section{ACM Reference Format:}

Xulong Tang, Ashutosh Pattnaik, Onur Kayiran, Adwait Jog, Mahmut Taylan Kandemir, and Chita Das. 2019. Quantifying Data Locality in Dynamic Parallelism in GPUs. In ACM SIGMETRICS / International Conference on Measurement and Modeling of Computer Systems (SIGMETRICS '19 Abstracts), June 24-28, 2019, Phoenix, AZ, USA. ACM, New York, NY, USA, 2 pages. https://doi.org/10.1145/3309697.3331473

\section{INTRODUCTION}

Dynamic Parallelism (DP) is a feature supported by CUDA and OpenCL ${ }^{\mathrm{TM}}$. It allows the generation and scheduling (launching) of kernels dynamically on GPUs without the involvement of a host (CPU). This model of computation is quite useful for irregular applications with unstructured and irregular inputs, as it essentially

Permission to make digital or hard copies of part or all of this work for personal or classroom use is granted without fee provided that copies are not made or distributed for profit or commercial advantage and that copies bear this notice and the full citation on the first page. Copyrights for third-party components of this work must be honored. For all other uses, contact the owner/author(s).

SIGMETRICS '19 Abstracts, fune 24-28, 2019, Phoenix, AZ, USA

(C) 2019 Copyright held by the owner/author(s).

ACM ISBN 978-1-4503-6678-6/19/06.

https://doi.org/10.1145/3309697.3331473 increases parallelism on-the-fly [1]. Specifically, if there are threads which are more compute-intensive than other threads, then these threads (parent threads) can parallelize their work by launching more threads (child kernels). This would allow for better (and ondemand) load balancing as there are higher number of threads to distribute across the GPU cores. However, by increasing the parallelism and redistributing the threads, the data reuse and access pattern can change dramatically. For example, original intra-thread temporal data reuse (i.e., the data blocks that are reused within a thread) can translate to inter-thread temporal reuse, due to the fact that parent thread offloads computations to its child threads. Moreover, this intra-thread temporal data reuse can even translate to inter-thread spatial locality, as the child kernel has multiple threads, and each child thread can work on a small portion of data.

Our goal in this paper is three-fold: (1) to quantitatively analyze the intrinsic data reuse opportunities in DP applications; (2) to reveal the best achievable performance improvements through a limit study; and (3) to propose a practical scheduling mechanism that improves data locality. To this end, we first conduct an in-depth data reuse analysis. We define a new "metric" called reuse ratio which captures the "intensity" of data reuse. The reuse ratio is computed for each pair of "schedulable units" where a schedulable unit is either a kernel, a work-group, or a wavefront. We then perform a limit study that exploits the reuse ratios for all possible permutations of the schedulable units at each level of the scheduling hierarchy and optimizes the placement (temporally and spatially) of the schedulable units to maximize data locality. Finally, we modify the hardware schedulers and propose a practical scheduling mechanism that schedules the schedulable units in a locality-aware fashion. This paper makes the following major contributions:

- It provides an in-depth data reuse quantification and analysis of GPU dynamic parallelism applications. The analysis is at multiple granularities (kernel, work-group, and wavefront) with respect to suitability for improving cache locality via scheduling techniques.

- It defines a new metric called reuse ratio, which captures the intensity of data reuse among schedulable units. We discuss the merits of this metric and demonstrate how to use it to guide scheduling strategies for DP applications.

- It performs a limit study by proposing an optimal scheduling mechanism that optimizes compute placement for cache locality by exploiting reuse ratios at each level of scheduling, viz. kernel, work-group and wavefront. This is achieved by providing accurate "reuse ratio" information to the hardware schedulers. The 
optimal scheduler provides, on an average, $19.4 \%$ performance improvement over the baseline scheduler.

- Based on the key observations from the data reuse analysis, it proposes LASER, a Locality-Aware SchedulER, that monitors the reuse ratio metric and makes scheduling decisions based on it. Our experimental results show that, on an average, LASER provides $11.3 \%$ performance improvement over the baseline scheduler.

To our knowledge, this is the first work that systematically investigates the data reuse and access patterns of DP applications at various granularities of schedulable units. Our work is most closely related to the work of Wang et al. [2]. However, their approach does not differentiate between the children of a given parent kernel, nor does it consider the intra-kernel data reuse. Therefore, we quantify the memory behavior of DP applications in detail and analyze their data reuse patterns to answer the following key questions. Question 1: How prevalent are intra-kernel and inter-kernel data reuses in DP applications? Question 2: What is the effect of launch overhead on data reuse patterns? Question 3: Do neighboring workgroups have more of temporal or spatial reuse? and Question 4: Is it necessary to always prioritize the child work-groups?

\section{REUSE CHARACTERIZATION}

In this section, we conduct an in-depth characterization of data reuses at different schedulable units to answer the aforementioned four questions. Our observations are follows:

At kernel granularity, DP applications launch massive numbers of kernels (children). In addition to the data blocks being reused within a kernel, these light-weight child kernels exhibit high data reuses with their parent kernels as well as among themselves. However, long reuse distances prevent the underlying GPU caches from taking advantage of most reused data blocks. Our results further show that DP applications introduce parent-child and sibling-sibling kernel relationships in addition to the self-kernel relationship. The reuse distance analysis indicates that GPU cache system is unable to take advantage of inherent data reuses in DP applications. We define and analyze reuse ratio to indicate the "intensity" of the data reuses among kernels, which is used later to design a locality-aware kernel scheduling mechanism.

At work-group granularity, not only neighboring WGs significantly share data blocks, but also the parent-child WGs and siblingsibling WGs. This information is helpful in assisting WG-to-CU mapping. Specifically, WGs with high reuse ratios should be scheduled on the same CU and executed in close proximity in time, in order to take advantage of the per-CU L1 cache.

At wavefront granularity, DP applications have significant number of wavefronts exhibiting intensive intra-wavefront reuse. Moreover, there is also a sizable fraction of wavefronts showing intensive inter-wavefront reuse. Since a wavefront can offload its computations to other wavefronts by launching child kernels, some of the intra-wavefront data reuses are transferred to inter-wavefront data reuse between parent wavefront and child wavefronts.

\section{PROPOSAL AND EVALUATION}

We distill the observations and propose LASER, a Locality-Aware SchedulER, that makes scheduling decisions based on the reuse ratios. The reuse ratios are computed dynamically at runtime with minimal hardware overheads and no profiling requirements. We

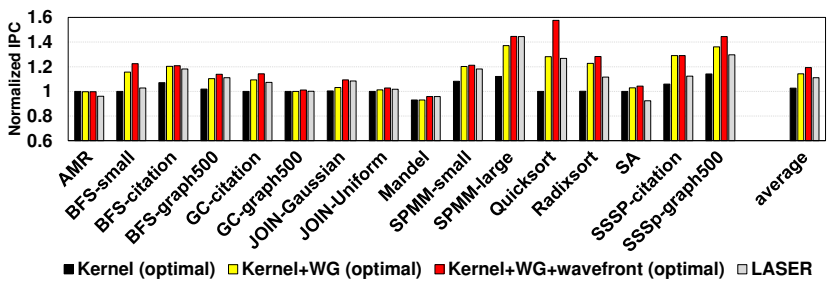

Figure 1: IPC normalized to baseline scheduling.

modify the baseline GPU architecture by extending/adding components in the GMU, WG scheduler, and CUs. Figure 1 show the normalized IPC across all 16 benchmarks. The results are normalized to the baseline scheduler which uses FCFS kernel scheduling, roundrobin WG scheduling, and GTO wavefront scheduling. The first three bars in Our limit study is a three-part study which involves an optimal locality-aware kernel scheduling policy(kernel), an optimal locality-aware kernel+WG scheduling policy (kernel+WG) and finally, an optimal locality-aware kernel+WG+wavefront scheduling (kernel+WG+wavefront). The last bar in Figure 1 shows the overall performance of LASER, normalized with respect to the baseline scheduler. LASER, on an average, achieves $11.3 \%$ performance improvement across the 16 GPU benchmarks tested.

\section{CONCLUSIONS}

Dynamic parallelism is an effective approach for improving GPU performance and resource utilization when executing irregular applications. While there have been prior efforts focusing on resource management and overhead tolerance for dynamic parallelism, the data access patterns and data reuse remain unclear. In this paper, we systematically characterize the data reuse and data locality opportunities that exist in dynamic parallel GPU applications. Based on our observations, we conduct a limit study to show the performance benefits of an "optimal" scheduler that realizes as much data reuse as possible. Furthermore, we propose a practical localityaware scheduler, called LASER, which makes the GPU hardware schedulers locality-aware, and thus improves data reuse. Our experimental evaluations show that, on an average, $19.4 \%$ and $11.3 \%$ performance improvements can be achieved with an optimal scheduler and LASER, respectively.

\section{ACKNOWLEDGMENT}

We thank Ganesh Ananthanarayanan for shepherding our paper. We also thank the anonymous reviewers for their constructive feedback. This research is supported in part by NSF grants \#1526750, \#1763681, \#1439057, \#1439021, \#1629129, \#1409095, \#1626251, \#1629915, $\# 1657336$ and \#1750667. AMD, the AMD Arrow logo, and combinations thereof are trademarks of Advanced Micro Devices, Inc. Other product names used in this publication are for identification purposes only and may be trademarks of their respective companies. OpenCL is a trademark of Apple Inc. used by permission by Khronos Group, Inc.

\section{REFERENCES}

[1] Xulong Tang, Ashutosh Pattnaik, Huaipan Jiang, Onur Kayiran, Adwait Jog, Sreepathi Pai, Mohamed Ibrahim, Mahmut Kandemir, and Chita Das. 2017. Controlled Kernel Launch for Dynamic Parallelism in GPUs. In HPCA.

[2] Jin Wang, Norm Rubin, Albert Sidelnik, and Sudhakar Yalamanchili. 2016. LaPerm: Locality Aware Scheduler for Dynamic Parallelism on GPUs. In ISCA. 